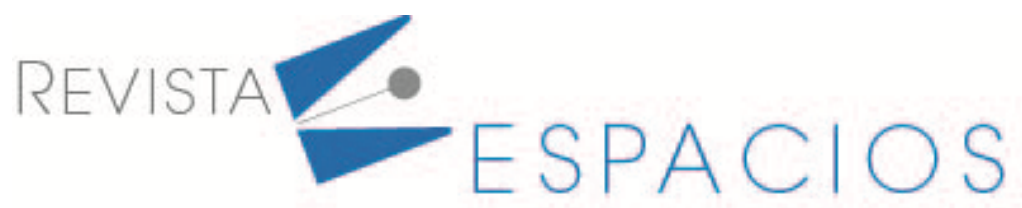

Vol. 42 (06) $2021 \cdot$ Art. 8

Recibido/Received: 12/02/2021 • Aprobado/Approved: 08/03/2021 • Publicado/Published: 30/03/2021

\title{
Os impactos da crise sanitária de COVID-19 sobre os Territórios Ultraperiféricos: $O$ caso de estudo do Arquipélago dos Açores (Portugal)
}

\section{The impacts of the COVID-19 health crisis on the Outermost Territories: The case study of the Azores Archipelago}

\author{
CASTANHO, Rui A. ${ }^{1}$ \\ COUTO, Gualter ${ }^{2}$ \\ PIMENTEL, Pedro 3 \\ CARVALHO, Célia ${ }^{4}$ \\ SOUSA, Áurea ${ }^{5}$ \\ BATISTA, María G. ${ }^{6}$ \\ GARRIDO-VELARDE, Jacinto 7,8
}

\begin{abstract}
Resumo
O presente estudo tornou evidente como a crise pandémica de COVID-19 afetou o turismo dos residentes nos Açores bem como as suas preferências e perceções. Desta forma, os autores acreditam que os dados obtidos através da investigação podem ser fundamentais para o adequado desenho e implementação de estratégias regionais para enfrentar a crise de SARS-COV-2. De facto, os resultados evidenciam as intenções das expectativas turísticas e as intenções dos residentes da Região Autónoma dos Açores, pelo que os resultados devem ser compreendidos como o reforço das medidas de segurança turística nas atividades turísticas de forma não só a estimular esta atividade mas também a relançar a economia e desenvolvimento regional.
\end{abstract}

Palavras-Chave: Região dos Açores; SARS-CoV-2; socio-economia; planeamento sustentável.

\begin{abstract}
The present study made it evident how the COVID-19 pandemic crisis affected the tourism of residents in the Azores as well as their preferences and perceptions. The results show the intentions of tourist expectations and the intentions of residents of the Autonomous Region of the Azores, so the results must be understood as the reinforcement of tourist security measures in tourist activities in order not only to stimulate this activity but also to relaunch the economy and regional development.
\end{abstract}

Key words: Azores region; SARS-CoV-2; socio-economy; sustainable planning.

\footnotetext{
${ }^{1}$ Faculdade de Economia e Gestão da Universidade dos Açores, Ponta Delgada, Portugal; e Faculdade de Ciências Aplicadas, Universidade WSB, Polónia acastanho@wsb.edu.pl

${ }^{2}$ Faculdade de Economia e Gestão da Universidade dos Açores e CEEApIA, Ponta Delgada, Portugal gualter.mm.couto@uac.pt

${ }^{3}$ Faculdade de Economia e Gestão da Universidade dos Açores e CEEApIA, Ponta Delgada, Portugal; pedro.ms.pimentel@uac.pt

${ }^{4}$ Faculdade de Ciências Sociais e Humanas da Universidade dos Açores e CINEICC, Ponta Delgada, Portugal; celia.mo.carvalho@uac.pt

${ }^{5}$ Faculdade de Ciências e Tecnologia da Universidade dos Açores e CEEAplA, Ponta Delgada, Portugal; aurea.st.sousa@uac.pt

${ }^{6}$ Faculdade de Economia e Gestão da Universidade dos Açores e CEEAplA, Ponta Delgada, Portugal; maria.gc.batista@uac.pt

${ }^{7}$ Department of Social Sciences, Languages and Literatures, University of Extremadura, 06071 Badajoz, Spain; jgarridoif@gmail.com // jgvelarde@unex.es 8 University Research Institute for Sustainable Territorial Development (INTERRA), University of Extremadura, Spain; jgarridoif@gmail.com // jgvelarde@unex.es
} 


\section{Introdução}

Ao longo da história, períodos de crise sempre foram precedidos por períodos de mudanças estruturais. Neste sentido, a atual crise sanitária, econômica e social que vivemos parece não ser uma exceção (ver estudos de: Abud, 2020; Couto et al., 2020; Gutiérrez, 2020). De entre as várias atividades afetadas pela pandemia de SARSCOV-2, o turismo parece ser uma das mais afetadas (corroborado por diversos estudos internacionais, como: Abu Bakar, and Rosbi, 2020; FAO, 2020; Mckibbin and Roshen, 2020; OECD, 2020; Ranasinghe et al., 2020; Sigala, 2020; UN, 2020); não apenas diretamente pelas viagens e voos cancelados, mas também pelo próprio medo de ser infetado pela doença (recomenda-se a leitura dos seguintes trabalho: Castanho, 2020; Espinoza, 2020; Mora Aliseda, 2020).

Neste sentido, é expectável que até ser encontrada uma vacina eficaz para o vírus, as estratégias mais eficazes para tentar travar o ritmo de contágio da doença são confinamentos em massa e medidas de distanciamento social. Ainda assim, as medidas e estratégias de confinamento nem sempre são fáceis de implementar. De facto, estas medidas apresentam-se de forma ainda mais complexa de serem implementadas em economias ligadas intrinsecamente à produção e à comercialização de bens e turismo (ver: CEPAL, 2020; Ramírez-Silva, 2020), como disso são exemplo os países africanos ou latino-americanos e sul-americanos como a África do Sul, o Brasil, o México, a Colômbia, o Perú, entre tantos outros.

A este respeito, torna-se evidente que o turismo foi a atividade mais afetada pela crise de COVID-19 (RamírezSilva, 2020). Efetivamente, o cancelamento de viagens aéreas associado ao medo de contrair o vírus parece ser um dos fatores mais relevantes, neste momento (Couto et al., 2020). Sensivelmente, a meados do mês de março de 2020, a maioria dos países declararam medidas fortes de distanciamento social, bem como da suspensão de todas as atividades não essenciais (Castanho, 2020). Essa a sociedade de uma forma transversal. Contudo, verificou-se um impacto mais evidente no turismo, uma vez que esta atividade foi totalmente interrompida (Ramírez-Silva, 2020).

Não obstante, diversos investigadores foram alertando ao longo das últimas décadas sobre a possibilidade de ocorrer uma crise sanitária em grande escala e consequentemente abalar a sociedade e o turismo em proporções nunca antes conhecidas, disso são exemplo os estudo de Gossling (2002), Hall (2006), Page e Yeoman (2007), Fauci e Morens ( 2012). Neste sentido os seguintes estudos poderão ser considerados como base para o acima exposto; da mesma forma os autores recomenda a sua leitura de forma a aprofundar a temática (ver: Scott e Gossling, 2015; Brown et al., 2016; Qureshi, 2016; Abukhalifeh et al., 2017; Rosselló, Santana-Gallego e Awan, 2017; Qiu et al., 2018; Bloom e Cadarette, 2019). Além disso, segundo as conclusões de Burkle (2006): “(...) as relações entre pandemias e viagens são fundamentais para compreender a segurança da saúde e a transformação global".

Face ao exposto, se consideramos territórios ultraperiféricos, como disso são o exemplo das regiões insulares, onde por norma, o turismo desempenha um papel fundamental na sua esfera socioeconómica (recomenda-se a leitura complementar dos seguintes estudos relativos a territórios periféricos: Couto, Pimentel e Ponte, 2017; Castanho et al., 2018; 2020; Lousada et al., 2028; Santos, Castanho e Lousada, 2019; 2020; Castanho, Couto e Pimentel, 2020; Naranjo Gómez et al., 2020). Logo, os autores acreditam que as consequências da crise de COVID-19 podem ser ainda mais evidentes nesta tipologia de territórios.

Contextualmente, o presente estudo pretende identificar e definir os impactos da crise de COVID-19 sobre o território ultraperiférico da Região Autónoma dos Açores através da análise das percepções dos residentes do Arquipélago dos Açores. 


\section{Materiais e Métodos}

Considerando o âmbito e intenção do presente estudo, os autores necessitaram de recorrer à utilização de diversas abordagens e técnicas de investigação, incluindo métodos de análise direta e indireta. De forma a simplificar o processo metodológico utilizado, este, pode ser sumarizado em quatro etapas principais:

- Etapa 1: recolha de dados, revisão bibliográfica e definição da oportunidade de investigação;

- Etapa 2: análise do estudo de caso, desenho e implementação de inquéritos de percepção pública;

- Etapa 3: delineamento e coordenação das equipas de investigação, e interpretação dos resultados;

- Etapa 4: discussões e conclusões - incidindo sobre a identificação e definição dos impactos causados pela crise de SARS-CoV-2 sobre o território ultraperiférico da Região Autónoma dos Açores.

\section{1. Área de Estudo}

A este respeito, o Arquipélago dos Açores foi utilizado como a área de estudo de caso da presente investigação. Baseado em estudos anteriores que a equipa de investigação já havia realizado sobre a temática de governança e gestão territorial na Região autónoma dos Açores, tais como os trabalhos de Vieira et al., 2014; Couto, Castanho, Couto e Pimentel, 2020; Castanho et al., 2020; Couto et al., 2020; foi possível obter conhecimentos prévios sobre esta realidade regional particular, permitindo-nos recolher uma quantidade e qualidade de dados, ainda, mais significante. Portanto, os autores obtiveram uma compreensão estratégica das problemáticas fundamentais relativas ao impacto da crise de COVID-19 sobre o território ultraperiférico da Região Autónoma dos Açores. Foi ainda possível compreender como a pandemia poderá afetar o desenvolvimento sustentável planeado para a região.

Os Açores apresentam um vasto potencial para o desenvolvimento desta tipologia de turismo. Em parte, tal potencial pode ser explicado pela sua localização estratégica. Além disso, a Região tem sido reconhecida como um destino sustentável baseado na natureza (ver: Castanho, Couto e Pimentel, 2020). Através da sua remota localização e natureza deslumbrante, foi várias vezes reconhecida, como turismo de aventura por referências internacionais, como disso são exemplo a Departures, a BBC, a Bloomberg, a Forbes, a GeekyExplorer ou o Lonely Planet. Adicionalmente, o património natural e cultural dos Açores, associado ao meio rural generalizado, fazem com que o Arquipélago tenha uma excelente localização para o desenvolvimento de aventuras de turismo rural e de natureza (ver: Ponte et al., 2018). Aqui, deve salientarse a liberalização parcial do transporte aéreo na Região em 2015, que influenciou a origem das operações das companhias aéreas de baixo custo (low-cost). Isto tem contribuído para o aumento do número de turistas e promovido uma variação significativa no comércio local (Couto, Pimentel e Ponte, 2017).

Na Região dos Açores, o turismo é visto como um setor de importância estratégica, uma vez que pode participar de forma decisiva no crescimento e desenvolvimento do Arquipélago (Vieira et al., 2014). Além disso, o turismo de aventura e de natureza são produtos de primeira linha deste modelo na Região (Couto, Pimentel e Ponte, 2017). Como consequência da nova dinâmica do setor turístico, surgiram mais atividades e empresas turísticas, aumentando e diversificando a oferta de experiências turísticas. Portanto, os Açores já apresentam algumas decisões práticas e administrativas que poderão facilitar o investimento nesta tipologia de turismo. Neste sentido, a atual janela de oportunidade criada pela crise de COVID-19 pode ser compreendida como o catalisador para a Região impulsionar este tipo de turismo sustentável. Nesta linha, a fim de incentivar as férias e as viagens dentro da Região, o governo dos Açores concebeu um incentivo às férias dentro do Arquipélago. O incentivo é exclusivamente dedicado aos residentes e cobre os serviços de transporte aéreo, alojamento, alimentação, atividades turísticas, aluguer de viatura e despesas de reserva em $50 \%$ do valor pago (até um limite máximo de 150,00 € por pessoa). 


\subsection{Inquéritos de perceção pública: desenho, amostra e tratamento de dados}

Considerando os requisitos de distanciamento social no cenário atual de crise pandémica, os inquéritos foram elaborados através da realização de entrevistas na web. A amostra foi constituída por participantes residentes da Região dos Açores e contou com mais de setecentos indivíduos. A amostra foi selecionada considerando que estes participantes seriam as principais fontes de conhecimento para a investigação proposta. Os inquéritos foram realizados na primavera de 2020 , durante os meses de maio e junho.

Após a recolha de dados resultantes dos questionários, foram aplicados métodos e ferramentas estatísticos. Numa primeira fase, foram aplicados métodos estatísticos através do Statistical Package for the Social Sciences (SPSS), atualmente designado por IBM SPSS Statistics.

\section{Resultados}

O questionário iniciou-se com uma primeira componente inerente às características sociodemográficas dos participantes (Tabelas 1 e 2). Desta forma, foi possível observar que a maioria dos participantes pertencia ao sexo feminino $(66,9 \%)$ e que a faixa etária mais representativa foi a referente aos 35 a 54 ou anos $(53,9 \%)$, precedida pela faixa etária dos 18 a 34 anos (24,0\%), e em última posição os participantes com 55 ou mais anos (22,1\%). Da mesma forma, evidenciou-se que $64,2 \%$ dos entrevistados estavam casados ou em situação de união de facto. Relativamente à situação profissional dos participantes a mais representativa identificou-se no pessoal superior (31,7\%), sendo a menos representativa os participantes em situação de desemprego (4,1\%). 0 grau acadêmico inquiridos com maior representatividade foi o Ensino Universitário $(58,1)$ e o menos representativo foi o Ensino Básico (5,1\%). Relativamente à Ilha de residência dos participantes (Tabela 2), foi possível verificar que a maioria deles são naturais e/ou residentes da llha de São Miguel (51,3\%); em sentido oposto, a Ilha com menor expressão de participantes no inquérito foi a llha do Corvo (0,3\%).

Tabela 1

Perfil dos inquiridos.

\begin{tabular}{|c|c|c|c|}
\hline \multicolumn{2}{|c|}{ Género } & \multicolumn{2}{|c|}{ Situação profissional } \\
\hline Feminino & $66,9 \%$ & Desempregado & $4,1 \%$ \\
\hline Masculino & $33,1 \%$ & Gestor & $8,1 \%$ \\
\hline \multicolumn{2}{|c|}{ Faixa etária } & Estudante/estagiário & $5,7 \%$ \\
\hline $18-34$ & $24,0 \%$ & $\begin{array}{c}\text { Trabalhador por } \\
\text { conta própria }\end{array}$ & $14,0 \%$ \\
\hline $35-54$ & $53,9 \%$ & Pessoal intermédio & $30,8 \%$ \\
\hline $55+$ & $22,1 \%$ & Pessoal superior & $31,7 \%$ \\
\hline \multicolumn{2}{|c|}{ Estado civil } & Reformado & $5,6 \%$ \\
\hline $\begin{array}{c}\text { Casado ou/em } \\
\text { comunhão de } \\
\text { facto }\end{array}$ & $64,2 \%$ & \multicolumn{2}{|c|}{ Grau académico } \\
\hline Divorciado & $10,3 \%$ & Ensino básico & $5,1 \%$ \\
\hline Solteiro & $23,4 \%$ & Ensino secundário & 36,75 \\
\hline Viúvo & $2,1 \%$ & $\begin{array}{c}\text { Ensino } \\
\text { universitário }\end{array}$ & $58,1 \%$ \\
\hline
\end{tabular}

Fonte: Autores 
Tabela 2

Ilha de residência.

\begin{tabular}{|c|c|}
\hline Ilha de residência & $\%$ \\
\hline Corvo & $0,3 \%$ \\
\hline Faial & $11,9 \%$ \\
\hline Flores & $1,6 \%$ \\
\hline Graciosa & $0,7 \%$ \\
\hline Pico & $5,0 \%$ \\
\hline Santa Maria & $3,3 \%$ \\
\hline São Jorge & $2,7 \%$ \\
\hline São Miguel & $51,1 \%$ \\
\hline Terceira & $23,3 \%$ \\
\hline
\end{tabular}

Fonte: Autores.

Nesse sentido, através da utilização de uma escala de avaliação de Likert (de cinco pontos), os participantes do estudo foram solicitados a declarar a sua concordância com doze afirmações, sendo que 1 representa uma discordância total com a afirmação e 5 uma concordância total com a afirmação. Após tratamento e análise dos dados recolhidos, as afirmações foram divididas em dois grupos: 1) iA a iF; e 2) iiA a iiF.

Os resultados apresentados na tabela 3 revelam que a moda referente ao grau de concordância com as afirmações dos itens i.A, i.B, i.C, e i.D foi concordo (4). Relativamente aos itens i.E e i.F, a os valores da moda foram 3 (um valor intermédio em relação ao nível de concordância) e 2 (discordo), respetivamente. 0 item com maior nível de concordância por parte dos participantes foi i.A, demonstrando que é seguro confiar no selo 'Covid-19 free' e/ou "Limpo e Seguro " atribuído pelas Autoridades de Saúde portuguesas, aos alojamentos turísticos. Por outro lado, o menor nível de concordância verificado foi vinculado ao item i.F. Neste sentido, resposta mais prevalente foi "Discordo" (2), o que indica algum otimismo por parte dos participantes sobre a situação atual da COVID -19. Se olharmos para os itens ii, o valor mais elevada da moda foi o relativo ao item ii.C "concordo totalmente" (5). Relativamente aos itens ii.E e ii.F, as respostas mais prevalentes foram em ambos casos "concordo" (4). No item ii.A, a seleção mais predominante foi "discordo totalmente" (1), revelando de forma distinta a previsão de menor gasto nas férias de 2020, em oposição a 2019. Além disso, no caso do item ii.B, a resposta mais comum foi "Discordo" (2).

Dada a natureza ordinal dos itens em análise, foi utilizado o coeficiente de correlação de Spearman (Tabela 4). Desta forma, foi possível identificar correlações positivas, e estatisticamente significativas ( $p<0,01$ ); entre essas correlações devem ser destacadas as maiores que 0,3 isto é as correlações entre os seguintes pares de itens: i.A e i.B ( $r s=0,475 ; p=0,000)$; i.C. e i.D ( $r s=0,797 ; p=0,000)$; i.C e i.E ( $r s=0,588 ; p=0,000)$; i.C e i.F ( $r s=$ $0,400 ; p=0,000)$; i.D e i.E ( $r s=0,653 ; p=0,000)$; i.D.e i.F ( $r s=0,396 ; p=0,000)$; i.E e i.F ( $r s=0,395 ; p=$ 0,000). Neste sentido, na Tabela 5 são destacadas correlações positivas e estatisticamente significativas entre ii.C e ii.F, bem como entre ii.D e ii.E. 
Tabela 3

Valores inerentes ao grau de concordância com cada uma das afirmações i.A a i.F e de ii.A e ii.F

\begin{tabular}{|c|c|c|c|c|c|c|}
\hline & i.A & i.B & i.C & i.D & i.E & i.F \\
\hline \multirow{2}{*}{$\begin{array}{l}\text { Níveis de } \\
\text { concordância }\end{array}$} & 4 & 4 & 4 & 4 & 3 & 2 \\
\hline & ii.A & ii.B & ii.C & ii.D & ii.E & ii.F \\
\hline $\begin{array}{l}\text { Níveis de } \\
\text { concordância }\end{array}$ & 1 & 2 & 5 & 3 & 4 & 4 \\
\hline \multicolumn{7}{|c|}{ Afirmações (i) } \\
\hline i.A & \multicolumn{6}{|c|}{$\begin{array}{l}\text { Vou confiar e escolher um alojamento turístico que tenha um selo de saúde e higiene como } \\
\text { 'Covid-19 free' ou 'Limpo e Seguro' " atribuído pelas Autoridades de Saúde portuguesas. }\end{array}$} \\
\hline i.B & \multicolumn{6}{|c|}{$\begin{array}{l}\text { Vou viajar tranquilo se o uso de máscaras e outras proteções individuais estiver generalizado } \\
\text { no destino. }\end{array}$} \\
\hline i.C & \multicolumn{6}{|c|}{ A COVID-19 veio demonstrar que devemos evitar destinos turísticos muito populares. } \\
\hline i.D & \multicolumn{6}{|c|}{ A COVID-19 veio demonstrar que devemos evitar férias em períodos de época alta. } \\
\hline i.E & \multicolumn{6}{|c|}{$\begin{array}{l}\text { A COVID-19 veio demonstrar que as melhores férias em mais seguras são no } \\
\text { campo/turismo rural. }\end{array}$} \\
\hline i.F & \multicolumn{6}{|c|}{ No future vou evitar viagens de avião. } \\
\hline \multicolumn{7}{|c|}{ Afirmações (ii) } \\
\hline ii.A & \multicolumn{6}{|c|}{ Vou gastar mais nas férias de 2020 do que nas de 2019.} \\
\hline i.iB & \multicolumn{6}{|c|}{ As férias de 2020 serão mais curtas do que as de 2019.} \\
\hline ii.C & \multicolumn{6}{|c|}{ Vou viajar menos para o estrangeiro em 2020, do que em 2019.} \\
\hline ii.D & \multicolumn{6}{|c|}{ Normalmente, escolho sempre o destino turístico mais barato. } \\
\hline ii.E & \multicolumn{6}{|c|}{ Geralmente, escolho voos mais baratos para poupar. } \\
\hline ii.F & \multicolumn{6}{|c|}{ Vou evitar viagens longas de avião. } \\
\hline
\end{tabular}

Fonte: Autores

\section{Quadro 4}

Coeficiente de correlação de Spearman relativo aos itens i.A. a i.F. **

A correlação é significativa ao nível de significância de 0.01 (2-tailed)

\begin{tabular}{|l|l|l|l|l|l|l|}
\hline \multicolumn{2}{|l}{ i.A } & i.B & i.D & \multicolumn{2}{l|}{ i.E } \\
\hline i.A & 1 & $0.475^{* *}$ & $0.153^{* *}$ & $0.124^{* *}$ & $0.130^{* *}$ & $-0,020$ \\
\hline i.B & & 1 & $0.112^{* *}$ & $0.126^{* *}$ & $0.167^{* *}$ & -0.014 \\
\hline i.C & & & 1 & $0.797^{* *}$ & $0.588^{* *}$ & $0.400^{* *}$ \\
\hline i.D & & & & 1 & $0.653^{* *}$ & $0,396^{* *}$ \\
\hline i.E & & & & & 1 & $0,395^{* *}$ \\
\hline i.F & & & & & & 1 \\
\hline
\end{tabular}

Fonte: Autores. 


\section{Quadro 5}

Coeficiente de correlação de Spearman relativo aos itens ii.A. a ii.F.

** A correlação é significativa ao nível de significância de 0.01 (2-tailed)

* A correlação é significativa ao nível de significância de 0.05 (2-tailed)

\begin{tabular}{|l|l|l|l|l|l|l|}
\hline & ii.A & ii.B & ii.C & ii.D & ii.E & i.F \\
\hline ii.A & 1 & 0.074 & $-0.176^{* *}$ & 0.027 & -0.035 & -0.011 \\
\hline ii.B & & 1 & $0.282^{* *}$ & 0.066 & $0.092^{*}$ & $0.132^{* *}$ \\
\hline ii.C & & & 1 & $0.146^{* *}$ & $0.233^{* *}$ & $0.405^{* *}$ \\
\hline ii.D & & & & 1 & $0.405^{* *}$ & $0.250^{* *}$ \\
\hline ii.E & & & & & 1 & $0.290^{* *}$ \\
\hline ii.F & & & & & & 1 \\
\hline
\end{tabular}

Fonte: Autores.

Além disso, foi colocada ainda uma questão de seleção de opções quanto ao tipo de alojamento de férias (Tabela 6), com três opções: a) alojamento turístico em meio rural; b) alojamento turístico em ambiente urbano com espaços verdes; e c) Alojamento turístico em ambiente urbano sem espaços verdes. Ainda que os resultados tenham sido próximos, a mais selecionada foi a opção b (alojamento turístico em meio urbano com espaços verdes) com 47,8\%, seguida da opção a (alojamento turístico em meio rural) com 43,8\%. A opção menos selecionada foi o alojamento turístico em ambiente urbano sem espaços verdes (c), com apenas $8,4 \%$.

Quadro 6

Opções de seleção.

\begin{tabular}{|c|c|c|c|c|c|}
\hline & Opções & Frequência & Percentagem & $\begin{array}{l}\text { Percentagem } \\
\text { Válida }\end{array}$ & $\begin{array}{l}\text { Percentagem } \\
\text { Acumulada }\end{array}$ \\
\hline \multirow[t]{4}{*}{ Válido } & $\begin{array}{l}\text { a) Alojamento } \\
\text { turístico em meio } \\
\text { rural }\end{array}$ & 272 & 38.6 & 43.8 & 43.8 \\
\hline & $\begin{array}{c}\text { b) Alojamento } \\
\text { turístico em } \\
\text { ambiente urbano } \\
\text { com espaços verdes }\end{array}$ & 297 & 42.2 & 47.8 & 91.6 \\
\hline & $\begin{array}{c}\text { c) Alojamento } \\
\text { turístico em } \\
\text { ambiente urbano } \\
\text { sem espaços verdes }\end{array}$ & 52 & 7.4 & 8.4 & 100.0 \\
\hline & Total & 621 & 88.2 & 100.0 & \multirow[t]{3}{*}{ - } \\
\hline Missing & 99 & 83 & 11.8 & - & \\
\hline & Total & 704 & 100.0 & - & \\
\hline
\end{tabular}

Fonte: Autores.

Do total dos inquiridos, 620 que selecionaram pelo menos uma das opções de resposta da tabela anterior, a maioria considerou que o selo "Limpo e Seguro" é fundamental na escolha do alojamento e restauração $(75,8 \%)$ e na escolha do destino a visitar $(50,5 \%)$ (Tabela 7$)$. Desta forma os resultados sugerem que devem ser feitos esforços nesse sentido. 


\section{Quadro 7}

O selo "Limpo e Seguro" é importante para si?

\begin{tabular}{|c|c|c|}
\hline Opções & $\begin{array}{c}\text { Número de } \\
\text { participantes }\end{array}$ & Percentagem \\
\hline $\begin{array}{c}\text { Na escolha do alojamento e } \\
\text { restauração. }\end{array}$ & 470 & $27.9 \%$ \\
\hline $\begin{array}{c}\text { Na escolha de atividades } \\
\text { turísticas. }\end{array}$ & 173 & $50.5 \%$ \\
\hline Na escolha do destino a visitar & 313 & $2 \%$ \\
\hline
\end{tabular}

Fonte: Autores.

Foi ainda colocada uma questão de resposta fechada: Quais são seus planos de férias para 2020? (Tabela 8). A partir das respostas, foi possível destacar a resposta mais selecionada (57,5\%): Vou me proteger e passar as férias na minha residência habitual; $14,3 \%$ disseram que escolheriam um destino menos típico, 11,5\% disseram que não iriam tirar férias este ano (2020) e (16,7\%) escolheram a opção "Outra".

Quadro 8

Questão de resposta fechada: Quais

são seus planos de férias para 2020?

\begin{tabular}{|c|c|c|c|c|}
\hline Válido & Respostas & $\mathrm{n}$ & $\%$ & \% válida \\
\hline $\begin{array}{c}\text { 1. Vou me } \\
\text { proteger e passar } \\
\text { as férias na minha } \\
\text { residência } \\
\text { habitual. }\end{array}$ & $\begin{array}{c}\text { 2. Vou escolher } \\
\text { um destino menos } \\
\text { popular. }\end{array}$ & $9707 \%$ & $57.5 \%$ \\
\cline { 2 - 5 } & $\begin{array}{c}\text { 3. Não vou fazer } \\
\text { férias neste ano de } \\
2020 .\end{array}$ & 78 & $13.8 \%$ & $14.3 \%$ \\
\cline { 2 - 5 } & 4. Outra & 113 & $11.1 \%$ & $11.5 \%$ \\
\cline { 2 - 5 } & Total & 678 & $16.1 \%$ & $16.7 \%$ \\
\hline Missing & 99 & 26 & $96.3 \%$ & $100 \%$ \\
\hline
\end{tabular}

Fonte: Autores.

\section{Discussão}

Com base nestes resultados, torna-se evidente uma oportunidade de investimento em novos modelos de atividades de turismo rural no Arquipélago dos Açores. Neste sentido, diversos autores defendem a existência de um potencial significativo para o desenvolvimento e crescimento do turismo rural e de natureza na Região dos Açores (ver: Silva, e Almeida, 2013; Vieira et al., 2014; Couto, Pimentel e Ponte, 2017; Castanho et al., 2020). Além disso, essa tipologia de turismo é reconhecida por vários académicos como uma forma de obter o desenvolvimento sustentável regional (recomenda-se a leitura complementar dos seguintes estudos relativos a desenvolvimento regional sustentável: Mahony e Zyl, 2002; Labrianidis e Thanassis, 2003; Buckley, 2007; Morais et al., 2019; Santos, Castanho , e Lousada, 2019; 2020; ou Ulucak, Yücel e Koçak, 2019). Desta forma, os decisores e principais atores regionais deveriam aproveitar esta oportunidade criada pela crise pandémica de COVID-19 para projetar uma perspetiva mais sustentável para o Arquipélago.

Contextualmente, os resultados do estudo corroboram a posição dos autores, sendo que mais de $30 \%$ dos participantes inquiridos no estudo afirmaram que escolheriam outra (s) Ilha (s) da Região dos Açores para passar 
férias (diferente daquelas onde têm residência). Também, mais de $25 \%$ declaram que pretendem ficar na Região dos Açores nas suas férias e menos de $5 \%$ afirmam claramente que vão ficar na llha de residência. Face ao exposto, verificou-se que os participantes consideram destinos turísticos não populares como o campo evidenciando, uma vez mais, a relevância do turismo rural neste período de crise sanitária.

Embora o potencial territorial para desenvolver esta tipologia de turismo sustentável esteja presente, as empresas de turismo locais e regionais devem alterar e redesenhar algumas das suas estratégias e abordagens. Neste sentido, o estudo demonstra que os residentes do Arquipélago dos Açores se sentem mais seguros quando o alojamento é classificado como 'Covid-19 free' e/ou 'Limpo e Seguro' atribuído pelas Autoridades de Saúde Portuguesas. Além disso, a investigação também permite evidenciar que os turistas se sentem mais interessados em destinos onde o uso de máscaras e outras proteções individuais é generalizado.

De facto, o turismo interno sempre foi a salvação de destinos turísticos que sofrem choques externos e instantâneos negativos na demanda externa de turistas internacionais (Couto et al., 2020). No entanto, a necessidade de utilizar o transporte aéreo para o turismo doméstico de Portugal Continental para a realidade das ilhas tornou-se uma condição muito prejudicial no atual contexto pandémico. Acresce ainda, a necessidade de testar o vírus antes de entrar no território insular dos Açores, e após a permanência de 6 dias na Região dos Açores.

Considerando a presente investigação, é possível comprovar que a crise sanitária de COVID-19 transformou consideravelmente o paradigma das atitudes e perceções dos residentes da Região Autónoma dos Açores na determinação da época e tipologia das férias. Portanto, os resultados deste estudo devem ser considerados pelas autoridades regionais (atores principais e tomadores de decisão) a fim de produzir e executar com precisão as políticas públicas regionais de forma a enfrentar o surto de SARS-CoV-2. Além disso, os resultados da investigação devem ser interpretados para aprimorar os modelos de segurança do turismo nas atividades turísticas regionais. Logo, se tais diretrizes forem consideradas, é esperado um estímulo significativo à economia e ao desenvolvimento regional no médio-longo prazo.

\section{Conclusão}

No decorrer desta investigação, torna-se evidente que as intenções turísticas dos residentes do Arquipélago dos Açores foram consideravelmente afetadas pelo surto de COVID-19. Disso, é exemplo o cancelamento da viagem de férias por mais de $40 \%$ dos inquiridos, e os $37,8 \%$ que desistiram de viajar para fora do arquipélago. Ainda, mais de $20 \%$ dos inquiridos alteraram o destino das férias e mais de $35 \%$ modificaram o seu período de férias.

Ainda, uma percentagem considerável dos residentes da Região Autónoma dos Açores afirmou que se iria proteger e passar as férias na sua residência habitual, ficando claro o impacto da pandemia nas suas intenções turísticas. A estas evidências, acresce ainda que, cerca de $70 \%$ dos residentes na Região dos Açores afirmam que vão passar as férias de 2020 na Região.

Tais resultados, acarretam impactos massivos para os territórios, ainda mais para territórios insulares, onde a sua base socioeconómica está em grande parte dependente da atividade turística. Portanto, neste período tão específico e peculiar da história contemporânea, os decisores políticos e atores principais destes territórios devem abordar estrategicamente o planeamento do território bem como o desenho e implementação de políticas públicas, pois nesta fase o seu impacto (negativo ou positivo) será seguramente amplificado.

Embora a presente investigação expanda o nosso conhecimento e compreensão de como a crise pandémica de COVID-19 afetou as expectativas de turismo dos residentes nos Açores, persistem várias possibilidades para pesquisas futuras. A título de exemplo, este tópico abre uma janela de oportunidade para um estudo mais aprofundado e avaliação do potencial turístico dos Açores durante e após a crise de COVID-19, ou ainda para 
realizar um estudo comparativo da indústria do turismo regional e as intenções dos residentes regionais quanto às suas férias durante e após esta crise pandémica.

Além disso, a investigação foi realizada especificamente para a região dos Açores como um todo. No entanto, se fossem realizados estudos individuais em cada uma das nove ilhas do Arquipélago, o nosso conhecimento sobre esta questão poderia ser ampliado. Da mesma forma, se o estudo for replicado noutros territórios insulares, o nosso conhecimento relativo a esta temática também será enriquecido.

De facto, as estratégias territoriais (incluindo as políticas públicas regionais) mudam e transformam regiões, evidenciando a necessidade de processos de monitorização continuo e de novas investigações sobre a dinâmica e tendências dos fatores que poderiam influenciar tais mudanças territoriais. Nesse sentido, a atenção especial deve ser dada às perceções do público e à evolução da crise sanitária de COVID-19 desde diferentes perspetivas e campos temáticos.

\section{Agradecimentos}

Este trabalho é financiado por fundos nacionais através da FCT - Fundação para a Ciência e a Tecnologia, I.P., no âmbito do projeto UIDB/00685/2020.

\section{Referencias bibliográficas}

Abu Bakar, N., Rosbi, S. (2020). Effect of coronavirus disease (COVID-19) to tourism industry. International Journal of Advanced Engineering Research and Science. Retrieved from: 7. 189-193. 10.22161/ijaers.74.23.

Abud, D. (2020). Essay: Covid-19, Efects in the international trade of Rep. Dominican and other latin american countries. Special Issue of: Reflexiones sobre el coronavirus y sus impactos. Revista Científica Monfragüe Resiliente Scientific Journal, pp.26-29. ISSN:234-5457

Abukhalifeh, A., Faller, E. Ahmad, A., Tadros, S. (2017). Current Issue in tourism: diseases transformation as a potential risks for travellers. Global and Stochastic Analysis, 5. (7)

Bloom, D. E., Cadarette, D. (2019). Infectious disease threats in the 21st century: strengthening the global response. Frontiers in Immunology. 10, 549. Retrieved from: https://doi.org/10.3389/fimmu.2019.00549

Brown, A., Ahmad, S., Beck, C., Nguyen-Van-Tam, J. (2016). The roles of transportation and transportation hubs in the propagation of influenza and coronaviruses: a systematic review. Journal of Travel Medicine, 23(1), tav002. Retrieved from: https://doi.org/10.1093/jtm/tav002

Buckley, R. (2007). Adventure tourism products: price, duration, size, skill, remoteness. Tourism Management, 28(6): 1428-1433.

Burkle, F. M. (2006). Globalization and disasters: Issues of public health, state capacity and political action. Journal of International Affairs, 59(2), 231-265.

Castanho, R.A. (2020). A pandemic crisis shocking us all: the covid-19. Special Issue of: Reflexiones sobre el coronavirus y sus impactos. Revista Científica Monfragüe Resiliente Scientific Journal, pp.233-238. ISSN:234-5457

Castanho, R.A., Couto, G., Pimentel P.(2020). Principles of sustainable tourism and cultural management in rural and ultra-peripheral territories: extracting guidelines for its application in the Azores Archipelago. Cultural Management: Science and Education (CMSE) ISSN: 2512-6962 DOI: 10.30819/cmse.4-1.01 
Castanho, R.A., Couto, G., Pimentel, P., Carvalho, C., Sousa, A. (2020). Territorial management and governance, regional public policies and their relationship with tourism. A case study of the azores autonomous region. Sustainability, 12, 6059; doi:10.3390/su12156059

CEPAL - Comisión Económica para América Latina y el Caribe (2020). América latina y el caribe ante la pandemia del COVID-19. Retrieved from: https://repositorio.cepal.org/bitstream/handle/11362/45337/4/S2000264_es.pdf

Couto, G., Pimentel, P., and Ponte, J. (2017). Tourism development potential in an insular territory: the case of ribeira grande in the Azores. Journal of Tourism Research \& Hospitality, 6(2). doi: 10.4172/23248807.1000166

Couto, G., Castanho, R.A., Sousa, A., Pimentel, P., Santos, C., Carvalho, C. (2020). The impacts of COVID-19 crisis over the tourism expectations of the Azores Archipelago residents. Sustainability, 12, 6059; doi:10.3390/su12156059

Espinoza, D. (2020). La Propuesta de trabajo de Costa Rica para atender en la crisis pandémica por el SARSCOV-2 y la participación de las universidades estatales. Special Issue of: Reflexiones sobre el coronavirus y sus impactos. Revista Científica Monfragüe Resiliente Scientific Journal, pp.66-80. ISSN:234-5457

FAO (2020). The impacts of COVID-19 on the forest sector: how to respond? Rome Retrieved from: https://doi.org/10.4060/ca8844en.

Fauci, A. S., Morens, D. M. (2012).The perpetual challenge of infectious diseases. New England Journal of Medicine, 366(5), 454-461. Retrieved from: https://doi.org/10.1056/NEJMra1108296

Gossling, S. (2002). Global environmental consequences of tourism. Global Environmental Change, 12(4), 283302. Retrieved from: https://doi.org/10.1016/S0959-3780(02)00044-4

Gutiérrez, S. (2020). Essay: after the pandemic, mobility after Covid-19. Special Issue of: Reflexiones sobre el coronavirus y sus impactos. Revista Científica Monfragüe Resiliente Scientific Journal, pp.13-16. ISSN:2345457

Hall, C. M. (2006). Tourism, biodiversity and global environmental change. In S. Gossling \& C. M. Hall (Eds.), Tourism and global environmental change: Ecological, economic, social and political interrelationships (pp. 142-156). Routledge.

Labrianidis, L., Thanassis, K. (2003). A suggested typology of rural areas in Europe. Retrieved from: cordis.europa.eu (accessed 2 August 2020).

Lousada, S., Escórcio, P., Castanho, R., Loures, L., Naranjo Gómez, J.M., Fernández-Pozo, L., Mora Aliseda, J., Cabezas, J. (2018). A multi-variated analysis on ultra-peripheral territories. A case study approach: sustainability indicators proposal for Madeira Island. Chapter in the Book: Planeamiento sectorial: Recursos hídricos, espacio rural y fronteras. Ed. Thomson Reuteurs Aranzadi. pp. 293-303 ISBN 978-841309-065-8.

Mahony, K., Zyl, J. (2002). The impacts of tourism investment on rural communities: three case studies in south Africa. Development Southern Africa, 19 (1), pp. 83-103

Mckibbin W. and Roshen. F. (2020). The economic impact of COVID-19. In the Book: Economics in the Time of COVID-19. Publisher: Centre for Economic Policy Research. 
Mora Aliseda, J. (2020). El Coronavirus obliga a una respuesta sin fronteras. Special Issue of: Reflexiones sobre el coronavirus y sus impactos. Revista Científica Monfragüe Resiliente Scientific Journal, pp.6-8. ISSN:2345457

Morais, J., Castanho, R.A., Loures, L., Pinto-Gomes, C., Santos, P. (2019). Villagers' perceptions of tourism activities in lona national park: locality as a key factor in planning for sustainability. Sustainability, 11 , 4448; doi:10.3390/su11164448

Naranjo Gómez, J.M, Lousada, S., Velarde, J.G., Castanho, R.A., Loures, L. (2020). Land-use changes in the canary archipelago using the CORINE data: aretrospective analysis. Land, 9, 232.

OECD (2020). The impact of the coronavirus (COVID-19) crisis on development finance. Retrieved from: https://read.oecd-ilibrary.org/view/?ref=134_134569-xn1go1i113\&title=The-impact-of-the-coronavirus(COVID-19)-crisis-on-development-finance (Accessed on July 21st 2020).

Page, S., Yeoman, I. (2007). How visit Scotland prepared for a flu pandemic. Journal of Business Continuity \& Emergency Planning, 1(2), 167-182.

Ponte, J., Couto, G., Pimentel, P., Santos, C., Oliveira, A. (2018). Tourism activities and companies in a sustainable adventure tourism destination: the Azores. CEEApIA - Centro de Estudos de Economia Aplicada do Atlântico, Universidade dos Açores, Working Paper.

Qiu, W., Chu C., Mao, A., Wu, J. (2018) The Impacts on health, society, and economy of SARS and H7N9 outbreaks in China: a case comparison study. Journal of Environmental and Public Health, Retrieved from: https://doi.org/10.1155/2018/2710185

Qureshi, A. (2016). Economic and political impact of ebola virus disease. Chapter 13 in the Book: Ebola Virus Disease, Academic Press, 2016, Pages 177-191, ISBN 9780128042304, https://doi.org/10.1016/B978-0-12804230-4.00013-3.

Ramírez-Silva, J.P. (2020). Una óptica sustentable del turismo post Covid-19. Special Issue of: Reflexiones sobre el coronavirus y sus impactos. . Revista Científica Monfragüe Resiliente Scientific Journal, pp.104-112. ISSN:234-5457

Ranasinghe, R., Damunupola, A., Wijesundara, W., Karunarathne, C., Nawarathna, D., Gamage, S., Ranaweera, A., Idroos, A. (2020). Tourism after corona: impacts of Covid 19 pandemic and way forward for tourism, hotel and mice industry in Sri Lanka. https://doi.org/10.13140/RG.2.2.27955.17442.

Rosselló, J., Santana-Gallego, M., Awan, W. (2017). Infectious disease risk and international tourism demand, Health Policy and Planning, Volume 32, Issue 4, May 2017, Pages 538-548, https://doi.org/10.1093/heapol/czw177

Santos, R., Castanho, R.A., Lousada, S. (2019). Return migration and tourism sustainability in Portugal: extracting opportunities for sustainable common planning in Southern Europe. Sustainability, 11, 6468; doi:10.3390/su11226468

Santos, R., Castanho, R.A., Lousada, S. (2020). The portuguese emigrants' return and the impacts over tourism development in rural areas: directions for a sustainable planning. Chapter 5 (pp.85-100). in the Book: Espacios y Sociedades en Transformación. Ed. Thomson Reuters Aranzadi - Spain. ISBN 978-84-1346-6934. 
Scott, D., Gosssling, S. (2015). What could the next 40 years hold for global tourism? Tourism Recreation Research, 40(3), 269-285. https://doi.org/10.1080/02508281.2015.1075739

Sigala M. (2020). Tourism and COVID-19: impacts and implications for advancing and resetting industry and research. Journal of business research, 117, 312-321. https://doi.org/10.1016/j.jbusres.2020.06.015

Silva, F., Almeida, M. (2013). Sustentabilidade do turismo na natureza nos Açores - o caso do canyoning. Em Almeida, M. (Ed.), Turismo e desporto na natureza, 5-19, Estoril: Associação de Desportos de Aventura Desnível.

UN (2020). Covid-19 and tourism: assessing the economic consequences. Retrieved from: https://unctad.org/en/PublicationsLibrary/ditcinf2020d3_en.pdf (Acceded at August 31, 2020)

Ulucak, R., Yücel, A., Koçak, E. (2019). The process of sustainability: from past to present. Environmental Kuznets Curve (EKC)

Vieira, J., Couto, G., Pimentel, P., Menezes, A., Moniz, A., Sousa, F. (2014). The satisfaction of the nordic tourist with the Azores as a destination. Scandinavian Journal of Hospitality and Tourism, 13(1), 59-73. doi: $10.1080 / 15022250.2014 .959806$

Esta obra está bajo una Licencia Creative Commons

Attribución-NoCommercial 4.0 International

(cc) EY-NC 